\title{
Téoros
}

Revue de recherche en tourisme

\section{Conservation de la nature et tourisme d'aventure dans les communautés limitrophes du parc Saguenay}

\section{Elsa Beaulieu, Christiane Gagnon et François Guillot}

Volume 19, numéro 1, printemps 2000

Pour une culture du tourisme au Saguenay-Lac-Saint-Jean

URI : https://id.erudit.org/iderudit/1071802ar

DOI : https://doi.org/10.7202/1071802ar

Aller au sommaire du numéro

Éditeur(s)

Université du Québec à Montréal

ISSN

0712-8657 (imprimé)

1923-2705 (numérique)

Découvrir la revue

Citer cet article

Beaulieu, E., Gagnon, C. \& Guillot, F. (2000). Conservation de la nature et tourisme d'aventure dans les communautés limitrophes du parc Saguenay. Téoros, 19(1), 37-42. https://doi.org/10.7202/1071802ar 


\title{
Conservation de la nature ET TOURISME D'AVENTURE DANS LES COMMUNAUTÉS LIMITROPHES DU PARC SAGUENAY
}

\author{
Elsa Beaulieu et Christiane Gagnon \\ avec la collaboration de François Guillot
}

Les parcs nationaux de conservation ont comme fonction principale de préserver des espaces fragiles ou représentatifs d'écosystèmes, tout en les rendant accessibles à l'ensemble de la population. Cette mission les rend incompatibles avec les usages traditionnels du territoire et affecte la vie des communautés locales qui perdent ainsi la maîtrise de leur territoire et d'une partie des usages qu'ils en faisaient ou qu'ils auraient pu en faire. En contrepartie, les parcs font valoir leur potentiel de création d'emplois et de développement de l'industrie touristique locale. Dix-sept ans après l'implantation du parc Saguenay, la communauté de Rivière-Éternité attend toujours le plein emploi que le parc leur avait promis. Pourtant, depuis le début des années 1990, il y a bel et bien eu des initiatives de développement touristique local, notamment sur le plan du tourisme d'aventure. Après avoir fait un bilan des conséquences de l'implantation du parc Saguenay, le présent article examine l'expérience partenariale tripartite parcentreprise privée - OSBL de Rivière-Éternité en matière de tourisme d'aventure et propose des pistes de réflexion sur l'utilisation du tourisme d'aventure pour susciter de nouvelles formes de développement social dans les communautés rurales dévitalisées.

\section{INTRODUCTION}

La région du Saguenay-Lac-Saint-Jean est une des régions du Québec les mieux dotées en parcs de conservation : le parc marin du Saguenay-Saint-Laurent, le parc du Saguenay, le parc des Monts Valin et le parc de la Pointe-Taillon. Leur mission est « d'assurer la conservation et la protection permanente de territoires représentatifs des régions naturelles du Québec ou des sites naturels à caractère exceptionnel, tout en les rendant accessibles au public pour des fins d'éducation et de récréation extensive ${ }^{1} »$. Cette mission se concilie avec certaines activités récréatives de plein air dont : l'observation de la faune et de la flore, la randonnée pédestre, le ski de fond, le kayak de mer.

Si la venue d'un parc de conservation favorise la pratique de telles activités, grâce à des aménagements souples qui garantissent la protection de l'environnement, elle remet en cause les activités locales traditionnelles. En effet, 1'implantation des parcs de conservation peut entraîner la perte de certains usages par les communautés locales : villégiature, activités de prélèvement telles que la chasse ou la pêche, utilisation spontanée de certains lieux sociaux et identitaires (quais, grèves).

Par ailleurs, pour obtenir l'acceptation de leur projet par les collectivités territoriales voisines, les parcs font valoir le potentiel d'emplois, voire de croissance économique que peut susciter le projet, notamment en servant de produit d'appel touristique. Dans le cas du parc Saguenay, les communautés locales, en particulier Rivière-Éternité, ont cédé une partie de leur territoire en échange de promesses de retombées économiques. Les parcs de conservation et, avec eux l'industrie touristique, favoriseraient une diversification de l'économie locale auparavant axée sur l'exploitation des ressources forestières ou halieutiques. Le développement de l'industrie récréotouristique, notamment du tourisme vert, apparaissait ainsi le leitmotiv salvateur d'économie locale en reconversion. Dès lors, chaque collectivité territoriale rêvait de voir arriver des hordes d'investissements et de touristes. Conséquemment, la préservation du patrimoine naturel et culturel des communautés devait aussi, selon les promoteurs d'espaces protégés, favoriser la rétention des populations locales (Barabé, 1995).

Bien que la conservation de la nature soit en soi un but louable, il importe maintenant de se demander, d'abord, si les parcs ont rempli leurs engagements envers les communautés concernées et, ensuite, si le développement touristique se conjugue de facto avec le développement local viable.

Pour explorer brièvement cette problématique $^{2}$ touchant l'implantation des parcs Saguenay et Saguenay-St-Laurent et les 17 communautés limitrophes (figure 1), nous avons choisi, comme cas de figure, la communauté de Rivière-Éternité, porte d'entrée du parc Saguenay. Dans un premier temps, nous faisons une brève analyse diachronique (1981-1996) du développement social et économique de cette communauté locale, à partir de quelques indicateurs représentatifs. Dans un deuxième temps, nous examinons une expérience partenariale de développement touristique entre les principaux acteurs, soit le parc québécois, l'entreprise privée et les organisations sans but lucratif locales (OSBL). Enfin, à la lumière du cas de 
Rivière-Éternité, nous esquissons de nouvelles pistes de réflexion et d'action qui peuvent favoriser aussi bien la conservation de la nature que le développement social et économique des communautés locales, autrement dit un développement local viable.

\section{L'ÉVOLUTION DU DÉVELOPPEMENT DE RIVIĖRE-ÉTERNITÉ ET LA CRÉATION DU PARC SAGUENAY ${ }^{3}$}

\section{CONTEXTE HISTORIQUE DU RÉCRÉOTOURISME}

Tels les grains d'un chapelet, les maisons du village de Rivière-Éternité s'échelonnent tout au long de la route régionale 170. Le noyau du village se love au pied des montagnes. Quelque 600 âmes animent les lieux. Officiellement créée en 1974, c'est la plus jeune municipalité de la microrégion du Bas-Saguenay, célèbre pour sa statue juchée sur le cap Trinité du parc Saguenay qui attirait déjà, dès le début du siècle, des touristes américains endimanchés sur leurs beaux «bateaux blancs», coup d'envoi à une future vocation, le récréotourisme. Aujourd'hui, la communauté maintient ce lien particulier entre le tourisme et une des traditions de la religion catholique. En effet, au début de la saison estivale, il y a la messe et la bénédiction des bateaux et, au temps de la Nativité, chaque maison illumine son parterre d'une crèche de Noël.

Le potentiel touristique du secteur a amené, dès 1972, la Société de développement touristique de Rivière-Éternité à le protéger des coupes forestières et à acquérir les titres de propriété de la compagnie Price Brothers. Regroupant plus de 150 membres, la Société visait à préserver le caractère naturel du territoire, à conserver l'accès public au fjord et à mettre en valeur son important potentiel récréotouristique: «On a décidé qu'on n'enverrait pas nos arbres à l'extérieur, mais que ce serait les gens de l'extérieur qui viendraient ici pour voir nos arbres ${ }^{4} \gg$. Pendant près de dix ans, sous la supervision de la Société, l'ensemble de la population éternitoise a travaillé activement et bénévolement. Le nouvel accès terrestre et les aménagements réalisés par la communauté ont permis aux Éternitois de découvrir toute la beauté, voire la richesse de cette partie de leur territoire qui est même devenue un lieu prisé de loisirs et de rencontres : piquenique du dimanche, observation des bateaux remontant le Saguenay, pêche et navigation de plaisance.

\begin{tabular}{|l|c|c|c|c|}
\hline \multicolumn{5}{|c|}{ TABLEAU 1 } \\
Indicateurs socio-économiques de Rivière-Éternité, 1981-1996 \\
\hline INDicateur / ANNÉE & 1981 & 1986 & 1991 & 1996 \\
\hline Variation de la population (\%)* & $+2,8$ & $-2,4$ & $-4,8$ & $-6,4$ \\
\hline Population 15-44 ans (\%) & 50,8 & 50,4 & 51,2 & 33,9 \\
\hline Population 45 ans et plus (\%) & 15,9 & 19,1 & 21,5 & 26,1 \\
\hline Pop. ayant une faible scolarité (\%)** & 40,4 & 41,1 & 22,7 & 31,2 \\
\hline $\begin{array}{l}\text { Pop. ayant entrepris } \\
\text { des études universitaires (\%) }\end{array}$ & 1,1 & 0 & 4,5 & 3,2 \\
\hline Emploi/population (\%) & 45,7 & 46,7 & 47,7 & 41,3 \\
\hline Taux de chômage (\%) & 5,3 & 36,2 & 45,1 & 31,5 \\
\hline Familles à faible revenu (\%) & 13,8 & 29,8 & 18,8 & 28,6 \\
\hline Revenu moyen/ménage (\$) & 22686 & 24786 & 32671 & 33188 \\
\hline
\end{tabular}

*Par rapport au recensement statistique précédent.

** Moins d'une neuvième année d'études.

Source : Statistique Canada.

Entre-temps, les gouvernements ont élaboré leurs projets de tourisme dit social. L'éventuelle création d'un parc national, par Ottawa, aurait été accompagnée d'investissements substantiels (50 millions de dollars), créant ainsi un potentiel important d'emplois et d'achalandage (200 000 visiteurs/an). En pleine montée souverainiste, Québec a refusé de céder ses terres et a créé son parc du Saguenay (1983), projet de moindre envergure au niveau des investissements, notamment à cause du statut de conservation qui laissait présager moins de bénéfices financiers à l'échelle locale.

Le parc du Saguenay a développé son premier pôle à la baie Éternité et y a investi 6 millions de dollars sur une période de 10 ans (1983-1993) (Québec, n.d.). Le gouvernement a accordé 140800 \$ pour la construction de chalets locatifs aux limites du parc ; cela faisait partie d'une enveloppe de 10 millions de dollars, financée conjointement par Ottawa et Québec, pour soutenir des projets touristiques de la zone périphérique du parc.

En acceptant de céder son territoire pour des fins de protection de l'environnement, la communauté se privait d'un accès direct au fjord. En outre, la majeure partie de son territoire $(94 \%)$ échappait à son contrôle par la présence conjuguée du parc, d'une zec et de CAAF5 (Désy, 1996). Il s'agissait là d'une contrainte significative à tout développement local.

Selon l'étude de Gagnon et Fortin (2000), la communauté comptait intensément sur la mise en valeur du potentiel récréotouristique de son environnement, associé à la splendeur du fjord, afin de compenser la perte significative d'emplois liée à la restructuration de l'industrie forestière. Le passage d'un mode de développement axé sur le prélèvement de la ressource forestière vers celui de la conservation n'allait cependant pas de soi. La venue du parc du Saguenay et, par la suite, du parc marin Saguenay-Saint-Laurent, après avoir suscité de nombreux espoirs, n'a pas semblé avoir mené aux résultats attendus.

\section{QUELQUES INDICATEURS}

Plus de quinze ans après la création du parc du Saguenay, y a-t-il eu des changements observables dans le développement de la 
communauté ? Pour répondre à cette question, retenons les indicateurs suivants

- pour la sphère économique : taux de chômage, revenu moyen par ménage, emploi/population;

- pour la sphère sociale : variation de la population, poids de certains groupes d'âge, scolarité de la population, pourcentage de familles à faible revenu ${ }^{6}$.

En ce qui concerne l'économie locale, l'analyse révèle une situation économique peu enviable. En effet, alors qu' au début des années 1980 le taux de chômage est moins élevé que la moyenne régionale et le revenu moyen par ménage se compare avantageusement aux autres localités, très rapidement (notamment avec la récession), la situation se dégrade : le taux de chômage est en hausse de $40 \%$ durant la période 1986-1991 et il redescend légèrement à $31,5 \%$ en 1996 (tableau 1). De plus, la population continue de diminuer, soit $-6,4 \%$ de 1991 à 1996. Quant au rapport emploi/population, il connaît une certaine stabilité pendant dix ans pour chuter à $41,3 \%$ en 1996 . Un autre problème, commun au Québec à l'ensemble des pays industrialisés, affecte la communauté de Rivière-Éternité, le vieillissement de la population : en effet, les statistiques montrent une augmentation constante de la proportion de la population âgée de plus de 45 ans, de 15,9\% à 26,1\%. Quant à la proportion de la population active sur le marché du travail (15-44 ans), elle chute dramatiquement en 1996 pour n'atteindre que $33,9 \%$, ce qui confirme l'exode des jeunes et d'une partie des travailleurs actifs. Soulignons que l'indicateur revenu moyen par ménage est en hausse depuis 1981, ce qui est normal, mais qu'en comparaison avec la moyenne régionale, il y a un écart de -6000 \$ en 1991 (Statistique Canada, 1991). Du côté des bonnes nouvelles, notons une amélioration de la scolarisation de la population ${ }^{7},+9,2 \%$ durant la période 1986-1996, mais le nombre de personnes ayant entrepris des études universitaires (main-d'œuvre qualifiée) reste cependant très faible. Le tableau 1 ci-dessous donne une vue d'ensemble de l'évolution diachronique de ces indicateurs.

Si nous nous référons à la période initiale qui ici nous intéresse, soit 1983, année de la création du parc Saguenay, les indicateurs statistiques choisis ne font pas état

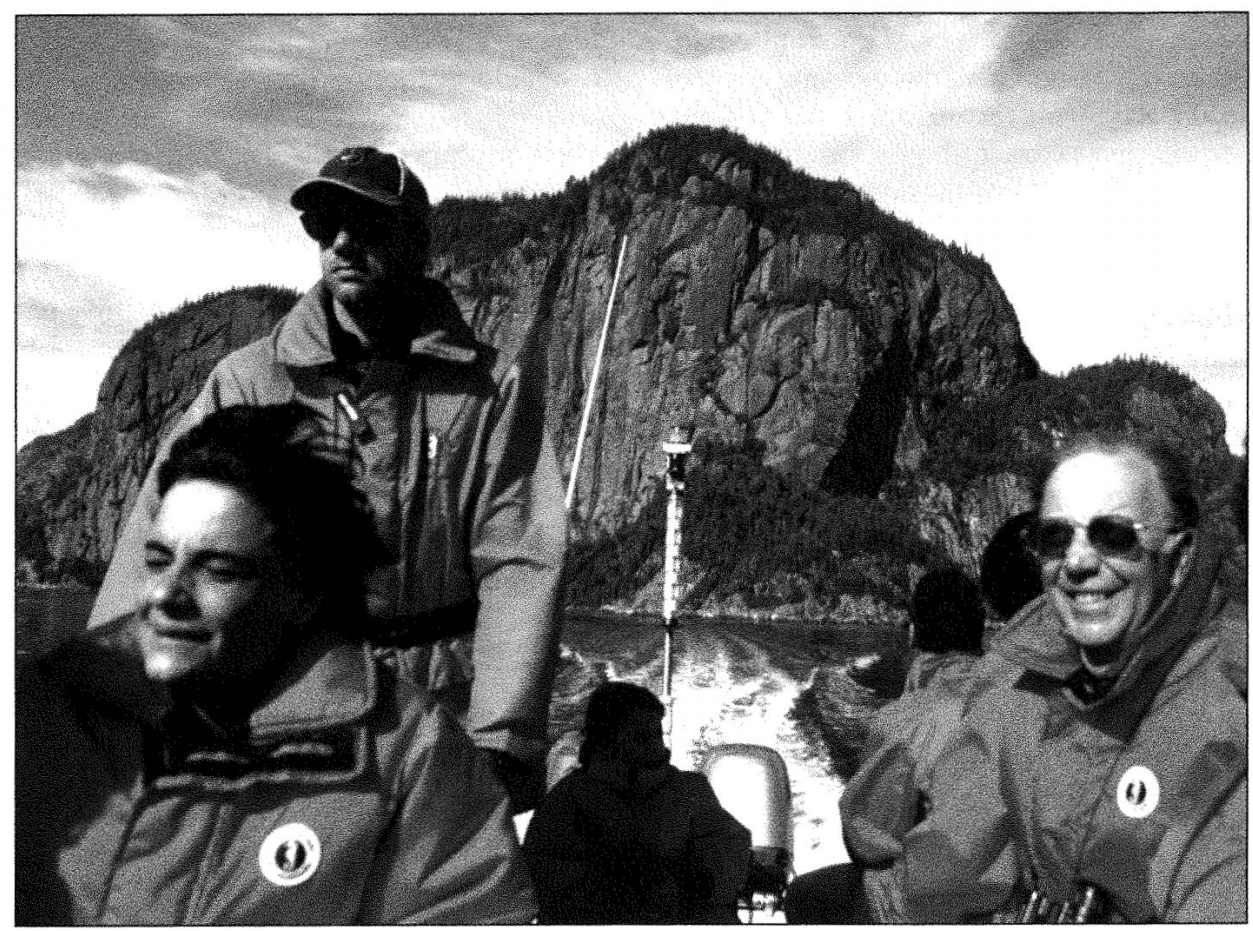

d'un changement qualitatif. Bien que les années 1980 aient vu une récession, ce qui affecte davantage les localités en difficulté, d'autres réussissent à tirer leur épingle du jeu. Il ne semble pas que l'arrivée du parc du Saguenay ainsi que des investissements gouvernementaux et privés (peu nombreux) aient modifié la direction du développement à Rivière-Éternité dans le sens d'un redressement positif.

Plusieurs explications peuvent être rattachées à cette situation, mais nous nous attardons ici à celles qui relèvent de l'industrie touristique. Tout d'abord, la fréquentation touristique est insuffisante : Rivière-Éternité reçoit, mal an bon an, la visite de plus ou moins 50000 personnes, concentrée sur quelques semaines estivales. Les emplois directs sont également très peu nombreux et saisonniers ${ }^{8}$. En fait, c'est surtout la Société de développement touristique qui crée le plus d'emplois locaux, soit une vingtaine. Afin de stimuler la fréquentation durant les autres saisons, la Société offre les produits de la pêche blanche et de la cabane à sucre éducative. L'idée la plus originale demeure sans doute celle de l'exposition intérieure des crèches de Noël couplée à celle des crèches mises en scène en façade des résidences privées. Cet élargissement des saisons touristiques et le développement de produits davantage enracinés dans la culture et les savoir-faire locaux semblent très prometteurs pour l'avenir de l'industrie récréotouristique.
Bien que cette industrie en soit encore à ses balbutiements, des leaders éternitois, forts de leurs dix années d'expérience, constatent que développement du tourisme demande un niveau élevé d'organisation : il y a nécessité d'obtenir des capitaux et des expertises. Il y a aussi nécessité de mobiliser les ressources et de favoriser la concertation entre les communautés du Bas-Saguenay de même qu'entre les organisations œuvrant sur le territoire ${ }^{9}$. Examinons de plus près, justement, une expérience partenariale liée au tourisme d'aventure.

\section{QUÉBEC HORS-CIRCUITS : L'EXPÉRIENCE D'UN TRIAN- GLE PARTENARIAL PORTEUR DE DÉVELOPPEMENT LOCAL}

\section{LES PARTENAIRES}

Le développement du tourisme d'aventure à Rivière-Éternité a été le fait de trois principaux partenaires: la Société de développement touristique de RivièreÉternité (SDTRE) au municipal, le parc du Saguenay (PS) au provincial et Québec Hors-Circuits inc. (QHC) au privé. La dynamique et l'organisation du triangle ainsi formé ont évolué dans le temps tout en conservant un objectif : le développement local de l'industrie touristique. Il est ainsi possible d'identifier, à partir de cette 
expérience, des éléments qui font obstacle au développement du tourisme d'aventure à Rivière-Éternité ainsi que des pistes de réflexion qui pourraient guider d'éventuelles interventions.

\section{CONTEXTE DE L'EXPÉRIENCE PARTENARIALE}

Le parc du Saguenay a été créé pour protéger les rives du fjord du Saguenay. Pendant plusieurs années, les activités commerciales du parc étaient gérées par le gouvernement provincial. Quelques postes ont été comblés localement, mais il s'agissait surtout de positions subalternes ou d'emplois de moindre importance (Fortin et Gagnon, 1999). Les postes spécialisés devaient souvent être comblés par des personnes de l'extérieur.

Quant à la participation des communautés locales aux processus décisionnels relatifs à la gestion du parc, elle n'a commencé à se développer de manière significative qu'au début des années 1990. Cependant, à partir de cette période, les budgets gouvernementaux consacrés aux parcs québécois ont commencé à diminuer progressivement. La stratégie utilisée par le parc du Saguenay pour pallier cette situation a été d'intéresser directement les communautés locales. À Rivière-Éternité, on a confié à la SDTRE l'exploitation ainsi qu'une partie du développement de tout le secteur compris entre Rivière-Éternité et Anse-Saint-Jean. De cette manière, il devenait possible de bénéficier de subventions fédérales et provinciales de toutes sortes pour soutenir le fonctionnement et le développement du parc, grâce au statut d'organisation sans but lucratif (OSBL) de la SDTRE. Ce partenariat bipartite permettait donc à la fois aux communautés locales de participer à l'orientation et à la gestion du développement et d'ouvrir la porte à d'autres sources de financement. Cette intervention nouvelle des communautés locales, qui leur assurait une maîtrise accrue de leur développement et de leur territoire, représentait un pas significatif en direction d'un développement local viable.

Vers le milieu des années 1990, la SDTRE a entrepris le développement d'activités de tourisme d'aventure, notamment le ski hors-piste, le kayak de mer et les excursions écotouristiques. Manquant de ressources humaines locales, la SDTRE a dû faire appel à une entreprise spécialisée dans le développement et l'exploitation de telles activités. C'est ainsi que la compagnie Québec Hors-Circuits inc. a été mandatée en tant que sous-traitante de la SDTRE. C'est à partir de ce moment que le partenariat bipartite s'est transformé en partenariat tripartite : un triangle dynamisé par l'expertise et l'audace commerciale d'une PME, structuré par l'intervention locale et encadré par la direction du parc. Au centre des préoccupations des trois partenaires, le développement de l'industrie touristique. Les principales forces de QHC étaient l'expertise, la compétence et des visions à long terme pour le développement, mais c'est le degré d'acceptation sociale élevée, dont l'entreprise bénéficie encore au sein de la communauté locale, qui a contribué avant tout à assurer son succès à l'intérieur du partenariat. Grâce à cette combinaison de facteurs, QHC a été amenée assez rapidement à prendre une place de plus en plus importante dans le développement et la gestion du secteur Rivière-Éternité du parc Saguenay ${ }^{10}$.

\section{LES OBSTACLES AU TOURISME D'AVENTURE ET LES CHANGEMENTS RÉCENTS}

Malgré des conditions favorables, le développement du tourisme d'aventure s'est heurté à deux obstacles importants : d'une part, les communautés environnantes disposaient de très peu de ressources humaines qualifiées pour les emplois spécialisés liés aux activités. D'autre part, les moyens financiers demeuraient limités, d'autant plus que les subventions gouvernementales se faisaient de plus en plus rares vers la fin de la décennie.

Cette situation de sous-financement des parcs était généralisée dans tout le Québec. Le gouvernement provincial envisageait alors de confier la gestion des parcs à un délégataire unique, pour en simplifier la gestion et résoudre les problèmes de financement ${ }^{11}$. En juillet 1999, la SÉPAC, société d'État déjà spécialisée dans le domaine, se voit chargée de l'opération des parcs québécois avec le mandat de les rentabiliser, grâce à une enveloppe budgétaire substantielle.

Le nouvel arrangement administratif devrait permettre de rénover les équipements désuets de certains parcs, de construire de nouvelles infrastructures, de rétablir l'uni- formité et l'image du réseau des parcs québécois ainsi que d'élaborer un plan de mise en marché. À court terme, il est probable que la création d'emplois soit stimulée. En effet, la SÉPAC s'est engagée à embaucher la main-d'œuvre locale lorsque possible ${ }^{12}$. Selon François Guillot, président de QHC et directeur général par intérim de la SDTRE Rivière-Éternité en 1998-1999, la venue de la SÉPAC est même synonyme de sauvetage aux yeux de certains villageois, c'est-à-dire de création d'emplois, tant la situation et le climat se sont détériorés au fil des ans. Toutefois, les Éternitois sont partagés sur cette question. Notamment, ceux qui se souviennent des efforts investis par la communauté locale dans le développement du potentiel touristique de Rivière-Éternité depuis 1972 ne voient pas nécessairement d'un bon œil la venue de la SÉPAC. Le nouveau mode de gestion par la SÉPAC influencera les orientations de développement local. En effet, avec la SÉPAQ les éternitois semblent leur faire craindre leur non-inclusion dans la gestion de l'espace protégé, malgré la promesse de création de tables d'harmonisation.

En outre, les décisions de la SÉPAC influenceront sans doute les entreprises locales du secteur touristique. Son slogan «partenaire en région» se traduira-t-il dans la réalité par un réel partenariat avec les PME et les OSBL locales? Il y a lieu de poser la question, car la SÉPAC semble davantage être employeur que partenaire pour l'entreprenariat local.

À la lumière de l'expérience de RivièreÉternité et des changements récents, deux constats retiennent notre attention. D'une part, il y a plus que jamais nécessité de former la main-d'œuvre locale de manière à ce que les communautés entourant les parcs puissent bénéficier des emplois créés par les parcs et par le produit d'appel touristique. D'autre part, il apparaît important que la SÉPAC favorise et valorise l'entrepreneuriat local tout en développant d'autres solutions, de concert avec la communauté.

\section{PISTES DE RÉFLEXION ET D'ACTION}

Comme nous l'avons mentionné, le manque de ressources humaines qualifiées constitue un obstacle au développement touristique et aux retombées locales de 
l'industrie. Cette question est d'autant plus complexe qu'elle est en relation avec des phénomènes tels que l'exode des jeunes et le décrochage scolaire qui, eux-mêmes, commandent des solutions originales pouvant mener à l'insertion sociale et l'intégration professionnelle des individus, notamment des jeunes. Nous avons vu dans le portrait de Rivière-Éternité que la population est vieillissante et fait face à un exode dramatique des jeunes qui partent pour étudier, mais qui ne peuvent ou ne veulent revenir dans leur milieu, faute d'emplois.

À cet égard, une étude réalisée en AbitibiTémiscamingue montre que l'insertion sociale des jeunes est préalable et nécessaire à leur intégration en emploi. De plus, des caractéristiques psychologiques telles que l'estime de soi jouent également un rôle important : «L'essentiel du processus d'intégration professionnelle passe par trois dimensions distinctes et complémentaires : l'estime de soi, l'éthique du travail et l'opportunité d'emploi. [...] La combinaison de ces trois facteurs favorise l'apparition d'autres éléments qui influencent à leur tour le processus d'intégration professionnelle» (Noreau, 1999). Une formation qui comprendrait des éléments favorisant l'apprentissage du leadership et le développement de la personne, en plus des compétences spécialisées, serait donc susceptible d'aider les individus à s'intégrer à la fois socialement et professionnellement.

Dans le domaine du tourisme d'aventure, une telle formation existe au SaguenayLac-Saint-Jean depuis 1996. En effet, le programme de baccalauréat en plein air et tourisme d'aventure, de l'Université du Québec à Chicoutimi, vise principalement à former des spécialistes du plein air pour œuvrer au sein de l'industrie du tourisme d'aventure, mais qui met aussi l'accent sur le développement de la personne. En outre, selon Mario Bilodeau, professeur à l'UQAC et spécialiste de l'éducation par le plein air et l'aventure, on peut utiliser l'éducation par l'aventure pour répondre à certains besoins sociaux des collectivités et des entreprises (Drouin, 1989).

Or, à Rivière-Éternité, en plus de vivre les phénomènes de chômage et d'exode des jeunes, typiques à beaucoup de régions rurales et forestières, la communauté s'est aussi vue dépossédée, comme nous l'avons mentionné dans la mise en contexte his-

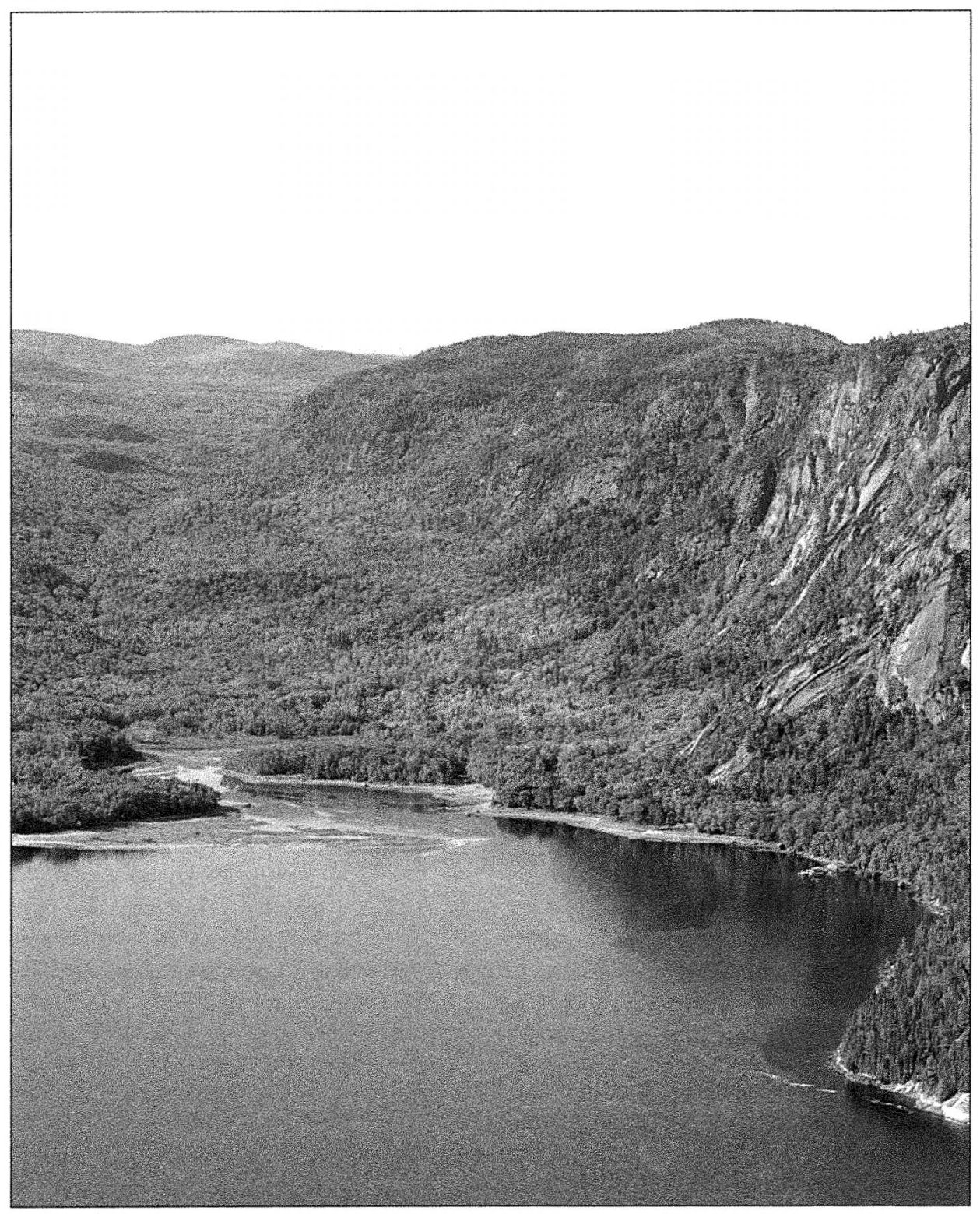

Rivière Éternité, photo Pierre Lahoud.

torique, du contrôle de la majeure partie de son territoire lors de l'implantation du parc. En conséquence, plusieurs citoyens vivent un sentiment de «désappropriation », de dépossession des lieux de vie (Fortin et Gagnon, 1999). La venue d'un parc de conservation et le développement du tourisme d'aventure, s'ils représentent théoriquement un potentiel de développement économique pour la localité, doivent par contre s'intégrer à la culture locale et contribuer à renforcer le tissu social de la communauté pour s'inscrire dans une démarche visant à promouvoir le développement local viable.

Cela est d'autant plus important que le tourisme d'aventure, tout comme la conservation de la nature, sont des pratiques qui, à l'échelle locale, présentent certaines discontinuités culturelles par rapport à l'utilisation traditionnelle du territoire. Il serait donc loisible d'imaginer que de jeunes diplômés, formés en tourisme d'aventure, pourraient s'intégrer dans une communauté rurale, en faisant le lien entre la culture locale et les nouveaux usages et pratiques liés à la nature. Ils pourraient ainsi contribuer au renforcement du sentiment d'appartenance au territoire, condition du développement local viable. La mise sur pied de tels programmes de formation s'avère une piste de développement du tourisme d'aventure, mais aussi une piste de valorisation d'activités récréatives et traditionnelles locales, donnant ainsi davantage de cohésion au développement social. 


\section{CONCLUSION}

Pour que la conservation des espaces protégés de même que le développement du tourisme soient viables localement, il faut que toutes les composantes sociales, culturelles, environnementales et économiques ainsi que les pratiques individuelles et collectives de maîtrise locale du territoire soient considérées de façon à répondre équitablement aux besoins fondamentaux, actuels et futurs, des communautés et de tous les groupes sociaux qui la composent, jeunes et moins jeunes. L'étude de cas de Rivière-Éternité révèle que les enjeux de la maîtrise du territoire et du partage des retombées économiques entre les acteurs et entre les communautés se trouvent au cœur du problème. Les stratégies de résolution appartiennent à chaque membre de la communauté, agent potentiel de changement, mais elles appartiennent également à tous les organismes publics ou privés qui tirent quelque avantage que se soit de l'environnement naturel ou bâti. L'injection de capital humain, dont celui de jeunes qui désireraient vivre dans les communautés rurales et contribuer à les repeupler, est sans aucun doute une voie d'avenir et de développement local viable. Non seulement des politiques publiques devraient-elles encourager cette piste d'action, mais concrètement, à court terme, les gestionnaires des espaces protégés pourraient tenter de favoriser ou même de susciter des initiatives en ce sens. À titre d'exemple, on pourrait penser à des bourses d'études ou des stages études-emploi offerts par la SÉPAQ ou Parcs Canada pour des jeunes désireux de relever le défi du développement social et de la conservation de la nature.

Une industrie récréotouristique florissante et une gestion intégrée des ressources ne sauraient se développer sans la participation éclairée et étroite des communautés locales concernées. Autrement dit, dans un contexte de ressources naturelles, tout projet de développement, tout aménagement et tout mode de gestion qui ne s'appuient pas sur des communautés en santé, qui ne valorisent pas l'empowerment des individus et des collectivités ainsi que la gouvernance environnementale locale (Gagnon et Fortin, 1999) ne peuvent prétendre à un succès à long terme.

Christiane Gagnon, Ph.D. en aménagement du territoire et spécialiste en évaluation des impacts, est professeure-chercheure à l'Université du Québec à Chicoutimi.

Elsa Beaulieu, bachelière en plein air et tourisme d'aventure, est présentement étudiante à la Maîtrise en études et interventions régionales à l'Université du Québec à Chicoutimi.

François Guillot est président de l'Association des producteurs en tourisme d'aventure du Québec, P.D.G. de Québec Hors-Circuits inc. et directeur des opérations à la pourvoirie du

\section{NOTES}

1 Loi québécoise sur les parcs, L.R.Q., c. P-9

2 Cette problématique a fait l'objet d'une recherche approfondie (1995-2000) dont l'argumentation et les résultats ne sont pas exposés dans cet article. Seule une étude de cas les illustre. Pour un exposé plus exhaustif, voir Fortin et Gagnon, 1999. Un rapport de recherche sera publié sous peu.

3 Cette partie, remise à jour, reprend globalement un texte publié dans le bulletin de l'INRS, Profils Saguenay (1997).

4 Entrevue avec M. Bertrand Bouchard, un des membres fondateurs de la société touristique.

5 Contrat d'approvisionnement et d'aménagement forestier (Ref. Loi 150 sur les forêts).

6 Certes, il ne s'agit là que d'un bref portrait. Pour une analyse en profondeur voir Gagnon et Fortin (2000).

7 N'oublions pas que ces proportions correspondent parfois à des petits nombres, + ou - 10 personnes, et que Statistique Canada, pour certains indicateurs, arrondit à plus ou moins 5 , ce qui peut entraîner une certaine inexactitude par rapport à la réalité.

8 Soit une quinzaine de postes saisonniers directement liés au parc, dont à peine le tiers est occupé par des Éternitois, les autres requérant des compétences spécialisées (naturalistes par exemple) que la main-d'œuvre locale ne possède pas et ce, sans compter la diminution du nombre de postes depuis quelques années en raison des compressions budgétaires.

9 Entrevues, 1996-1997.

10 Son président directeur général, François Guillot, a même agi à titre de directeur général par intérim de la SDTRE en 1998-1999.

11 Pour plus de détails, voir le Rapport du comité conseil sur la relance des parcs québécois, Ministère de l'Environnent et de la Faune (1996).

12 Entretien avec Jacques Bertrand, directeur du parc Saguenay.
Cap au Leste. Il a aussi été directeur général par intérim de la SDTRE en 1998-1999. Québec Hors-Circuits inc. est sous-traitante pour le parc Saguenay depuis 1996.

Un comité de lecture a lu et accepté ce texte

\section{BIBLIOGRAPHIE}

Barabé, André (1995), « Parc, tourisme et développement durable », Téoros, 14 (1) : 8-12.

DÉSY, Jean (sous la direction) (1995), Développement local et aménagement intégré du territoire et des ressources de Rivière-Éternité, Chicoutimi, Forespoir/GRIR, UQAC.

Drouin, Gilles (1989), « Le plein air, une question d'équilibre » (reportage réalisé à la suite d'entretiens avec Mario Bilodeau), Réseau, 20 (9).

Fortin, Marie-José, et Christiane Gagnon (1999), « An Assessment of Social Impacts of National Parks on Communities in Quebec, Canada », Environmental Conservation, 26 (3) : 200-211.

Fortin, Marie-José, et Christiane Gagnon (1997), « Rivière-Éternité, une communauté à la quête de la terre promise: le récréotourisme », Profils Saguenay, Bulletin de 1'INRS, 3 (1) : 6-7.

Gagnon, Christiane (sous la direction de) (1999), Tourisme viable, parcs nationaux, développement des communautés locales: quel avenir. Forum. Chicoutimi : GRIR/UQAC, 183 p.

Gagnon, Christiane, et Marie-José Fortin (à paraître), L'évaluation de la création des parcs nationaux sur le développement viable des communautés locales limitrophes : rapport de recherche, Chicoutimi : GRIR/UQAC.

Gagnon, Christiane, et Marie-José Fortin (1999), « Gouvernance environnementale locale : où est le timonier ? ", Économie et Solidarités, 30 (2) : 94-111.

Ministère de l'Environnent et de la Faune (1996), Rapport du comité conseil sur la relance des parcs québécois, Québec.

Ministère du Loisir, de la Chasse et de la Pêche (n.d.), Le parc du Saguenay et sa zone périphérique : 10 ans de vie active! Québec, $44 \mathrm{p}$.

Noreau, Pierre, et coll. (1999), L'insertion sociale et l'intégration professionnelle de jeunes : une recherche en Abitibi-Témiscamingue, Étude réalisée pour le CRCD A-T, à l'occasion de la table régionale jeunesse de l'AbibibiTémiscamingue. 